\title{
La OEA y la cooperación hemisférica
}

\author{
Pedro Oyarce*
}

Este documento desarrolla algunas reflexiones sobre las áreas de trabajo de la OEA vinculadas con las preocupaciones de nuestros ciudadanos: democracia, derechos humanos, seguridad multidimensional y desarrollo integral. Se trata de elementos esenciales para la gobernabilidad y la construcción de un Estado moderno, eficiente y transparente. La opinión pública y diversos sectores sociales plantean crecientes interrogantes que debemos considerar a fin de explicar el papel político de la Organización. Para evaluar con objetividad el desempeño de la OEA, es conveniente conocer algunos de sus aportes, avances y limitaciones. Debemos tener claro el carácter gubernamental de esta instancia y el desafío que tiene para consolidarse como un referente de cooperación en una región heterogénea. Es importante también considerar que la OEA es una organización intergubernamental que trabaja fundamentalmente por consenso.
En los últimos meses, especialmente a partir de la derogación de la Resolución de 1962 sobre $\mathrm{Cuba}^{1}$ y de la suspensión de Honduras ${ }^{2}$, hemos apreciado un renovado interés en analizar la capacidad de acción de la OEA. Este texto busca proporcionar elementos para ese propósito.

\section{Multilateralismo HeMisférico}

El multilateralismo es un factor determinante en las relaciones interamericanas y globales. A través de él los países pueden influir en los procesos de toma de decisiones y en la definición de regímenes e instituciones que buscan dar gobernabilidad a la región y al mundo. Entre las ideas que sus-

Embajador de Chile ante la OEA, Washington, D.C.

AG/RES. 2438 (XXXIX-O/09)

2 AG/RES. 2 (XXXVII-E/09) 
tentan esta afirmación, destacamos las siguientes que definen al Sistema Interamericano (SI):

1. La OEA es la depositaria de las normas de derecho internacional que han sido elaboradas en el marco regional. Es un espacio de reafirmación de principios y valores, tales como soberanía, no intervención, respeto del derecho internacional, solución pacífica de las controversias, protección de los derechos humanos y las libertades fundamentales, promoción y consolidación de la democracia y el Estado de derecho, buen gobierno, paz y seguridad.

2. Es un espacio de cooperación, en cuanto camino para potenciar las capacidades nacionales a fin de hacer frente a problemas compartidos.

3. Es un espacio para la elaboración de regímenes jurídicos, estándares internacionales y mecanismos modernos de seguimiento e implementación. Estos últimos incorporan un sistema de revisión entre pares, en virtud del cual los Estados evalúan periódicamente el cumplimiento de las obligaciones convencionales y recomiendan medidas correctivas cuando procedieren. Ejemplos de esta función son:

- El Mecanismo de Evaluación Multilateral de la Comisión Interamericana para el Control del Abuso de Drogas (MEM-CICAD). Es una modali- dad de implementación de las normas en el ámbito de la lucha contra las drogas.

- El Mecanismo de Evaluación y Seguimiento de la Convención Interamericana contra la Corrupción (MESICIC), que cumple un rol similar en un tema central para la democracia.

- El Mecanismo de Seguimiento de la Implementación de la Convención Interamericana para Prevenir, Sancionar y Erradicar la Violencia contra la Mujer, "Convención de Belém do Pará» (MESECVI).

- La Secretaría Técnica para el Desarrollo del Programa de Acción para el Decenio de las Américas por los Derechos y la Dignidad de las Personas con Discapacidad (2006-2016) (SEDISCAP). Tiene un papel análogo en materia de discapacidad.

4. Puede ser también un espacio de sanción. La OEA tiene capacidades limitadas en este ámbito y no dispone de competencias similares a las del Sistema de las Naciones Unidas en materia de medidas coercitivas.

\section{Multilateralismo Efectivo}

Este concepto tiene que ver con la exigencia de que el multilateralismo sea coherente en el plano sistémico. El SI es parte del sistema universal $(\mathrm{Na}-$ ciones Unidas). Los niveles globales y regionales son interdependientes y complementarios (Capítulo VIII de la 
Carta de las Naciones Unidas; acuerdos regionales previstos en el Art. 52). Se debe buscar un modelo multilateral operativo y eficaz, donde se eviten las duplicaciones y se favorezca la coordinación. Los esquemas regionales deben emplear sus ventajas comparativas socioculturales para implementar los principios y valores universales, no para relativizarlos.

Es probable que exista una tendencia hacia un multilateralismo más cooperativo, con mayores niveles de inclusión. El unilateralismo ha mostrado su ineficacia y la disposición de los Estados Unidos a trabajar colectivamente contribuye a una mirada más optimista respecto de la efectividad del sistema en temas regionales y globales.

Para el SI esto es clave y aunque se trata de una región diversa o heterogénea en múltiples ámbitos y con crecientes diferencias ideológicas, debemos continuar esforzándonos por preservar el multilateralismo hemisférico. La paz interestatal y social que hemos alcanzado es un capital político que debe mantenerse y en torno al cual deberíamos reconocer el aporte de un sistema regional articulado. Es necesario evitar que legítimos reagrupamientos limiten las capacidades y la acción de la OEA. El apoyo político debe ir acompañado de mayores recursos y esa es también una responsabilidad colectiva.

\section{DEMOCRACIA}

La contribución de la OEA en el área de la promoción y defensa de la democracia ha sido determinante desde la perspectiva normativa y política, con proyecciones culturales ${ }^{3}$. La Carta de la OEA contiene menciones concretas a la democracia (artículo 3). Diversas Resoluciones también lo hacen y la Quinta Reunión de Consulta de Ministros de Relaciones Exteriores, celebrada en Chile en 1959, identificó los elementos de la democracia representativa. Es útil comprender la importancia de ese esfuerzo en un contexto político muy distinto del actual.

En la acción de la OEA en esta área destacan algunos aspectos.

En primer lugar, un proceso de codificación del derecho a la democracia, que se ha expresado en las siguientes etapas:

1. La Resolución 1080 de 1990, presentada por Chile, que consagra la cláusula democrática. Es la primera ocasión en que se establecen sanciones para los países en los que se rompe el orden constitucional. Fue aplicada en Haití (1991). Como

3 En el período 2005-09, se han organizado 12 misiones para enfrentar crisis políticas e institucionales en los siguientes países miembros: Belice-Guatemala (2000-2008), Bolivia (2005 y 2008), Colombia-Ecuador (2008- actualidad), Colombia (2005- actualidad, 2007), Ecuador (2005), Guatemala (2000-2006), Haití (2000-2006, 2008), Honduras (2009), Nicaragua (2005) 
lo señala el Secretario de Asuntos Jurídicos de la OEA, Jean-Michel Arrighi, la crisis del Perú en 1992, en la que un gobierno legítimamente elegido disolvió el Congreso, y luego de gestiones de la OEA se convocó una Asamblea Constituyente, permitió advertir que era preciso complementar la Resolución 1080. Un problema central era que no se señalaban con precisión ${ }^{4}$ las modalidades que podían tener las interrupciones abruptas de la democracia. Este fue el inicio de un proceso que condujo a la aprobación de una Carta Democrática Interamericana.

2. El Protocolo de Washington de 1992 modificó la Carta de la OEA (artículo 9) e incorporó la cláusula democrática (suspensión en caso de quiebre democrático). Fue un avance, pero tampoco proporcionaba más elementos respecto de situaciones que no fueran el clásico golpe de Estado.

3. La Carta Democrática Interamericana (CDI), aprobada por Resolución de la Asamblea General de 2001 , no es formalmente un tratado, pero vincula a los países miembros de la OEA ya que desarrolla principios básicos de la Carta e intenta identificar distintas situaciones frente a un quiebre democrático. La relevancia de este instru-

4 Arrighi, Jean Michel (2008), «Derecho Internacional y Democracia en el Sistema Interamericano", Homenaje a Santiago Benadava; Santiago de Chile. mento rebasa el estricto valor jurídico de una resolución. La CDI es el mayor esfuerzo político del SI por promover la cooperación en esta esfera, la cual va más allá del análisis sobre la jerarquía y el alcance de estos instrumentos. Una corriente de opinión sostiene que se trataría de una costumbre regional, de un desarrollo normativo o de una interpretación de la Carta de la OEA, en especial de los artículos 2 b) y $3 \mathrm{~d})$. Este planteamiento sugeriría una excepción al principio de la no intervención, consagrado en el artículo 19 de dicha Carta, fundamentado en la legítima preocupación de la comunidad internacional frente a situaciones o crisis que amenacen la democracia. La tendencia muestra que invocar el principio de la no intervención para rechazar el interés en contribuir a la preservación de la democracia es hoy de difícil sustento. Esto tiene sentido especial en el contexto de las relaciones interamericanas.

En segundo lugar, la CDI ha implicado avances sustantivos, particularmente en los siguientes ámbitos:

1. Consagra el derecho de los pueblos a la democracia y la obligación de los gobiernos de promoverla y defenderla.

2. Reconoce que la democracia es esencial para el desarrollo social, político y económico de los pueblos. 
3. Establece los elementos constitutivos de la democracia representativa: 3.1. El respeto de los derechos humanos y las libertades fundamentales

3.2. El acceso al poder y su ejercicio con sumisión al Estado de derecho

3.3. La celebración de elecciones periódicas, libres, justas y basadas en el sufragio universal y secreto.

3.4. Un régimen plural de partidos y organizaciones políticas.

3.5. La separación e independencia de los poderes públicos.

La CDI distingue claramente dos conceptos: la democracia de origen (electoral) y la democracia de gestión (comportamiento democrático). Según este criterio no bastan las elecciones libres, secretas e informadas, que generan una legitimidad básica, sino que es necesario también gobernar democráticamente, respetando los elementos esenciales ya aludidos. Esto es lo que está en juego cuando se habla de una aplicación efectiva de la Carta.

La CDI describe situaciones que generan distintos niveles de respuesta para preservar una democracia amenazada o interrumpida en un Estado miembro.

Un análisis de diversos escenarios contenidos en ese documento permite constatar que «la Carta está hecha por gobiernos y para gobiernos».
ESCENARIO UNO (ART. 17). Un gobierno considera que está en riesgo:

1. El proceso político institucional

2. El legítimo ejercicio del poder

El Gobierno afectado solicita al Secretario General o al Consejo su asistencia para fortalecer y preservar la institucionalidad democrática. Se requiere entonces el consentimiento del país involucrado.

ESCENARIO DOS (ART. 18). En un Estado se producen situaciones que pudieran afectar:

1. El proceso político institucional

2. El legítimo ejercicio del poder

En este caso, la iniciativa de hacer gestiones, disponer visitas u otras medidas es del Secretario General o del Consejo. También se requiere el consentimiento previo del Gobierno afectado.

ESCENARIO TRES (ARTS. 19-2021). Contempla dos situaciones:

1. Una alteración que afecte gravemente el orden institucional o

2. La ruptura democrática.

En ambos casos se produce un obstáculo insuperable para que el gobierno pertinente participe en la Organización. Cualquier Estado o el Secretario General pueden pedir una apreciación colectiva en el Consejo Permanente, donde participan los gobiernos allí representados. Luego de agotadas las gestiones diplomáticas, puede darse lugar a la suspensión de dicho Estado en un período extraordinario de la Asamblea General. Esa decisión re- 
quiere de un quórum de dos tercios y desarrolla las normas contenidas en el artículo 9 de la Carta de la OEA, modificada por el Protocolo de Washington. Esto fue lo que ocurrió en el caso de Honduras en que se aplicó por primera vez el artículo 21 de la CDI. En esa ocasión, se adoptó la máxima sanción que contempla el SI (AG/RES. 2 [XXXVII-E/09]). Siempre la OEA, a través del Secretario General y de los Estados miembros, puede continuar haciendo gestiones diplomáticas para buscar una restauración de la democracia. Es lo que ha ocurrido con el Acuerdo de San José y las misiones de Cancilleres.

¿Cuáles son los desafíos que se plantean en la evaluación de las capacidades de la OEA? Frente a esta pregunta, formulamos tres observaciones:

1. En primer lugar, en los casos contemplados en la CDI operarían lo que denominaríamos «candados gubernamentales». Los Gobiernos en definitiva deciden la utilización de la Carta. Ellos son los únicos que participan en este proceso. Ya sea el gobierno que requiera asistencia de la OEA u otros, cuando se hace una apreciación colectiva y se deciden las gestiones o sanciones contempladas en la CDI. ${ }^{5}$

2. Este instrumento solo puede ser activado por el Ejecutivo y, en el hecho,

Sobre este punto véase Legler, Thomas (2007), La Carta Democrática: Retórica o Realidad Promoción de Democracia en las Américas, John Hopkins University Press. los países han rechazado la posibilidad de que sea invocada por otros poderes del Estado. El propio Secretario General sugirió al Consejo Permanente, en $2007^{6}$, una aplicación extensiva de la Carta, propuesta que planteó dificultades. Es evidente, entonces, que cuando otros Poderes del Estado se sientan afectados por acciones del Ejecutivo, no podrían poner en marcha los mecanismos de la Carta.

3. En tercer lugar, es necesario precisar qué se entiende por alteración que afecte gravemente el orden constitucional y cómo se define el obstáculo insuperable para la participación de un gobierno en la Organización. El ex Presidente Carter ha señalado situaciones que deberían incluirse en este concepto, las que desarrollan y complementan aquellas identificadas por la OEA en $1959^{7}$ e incorporan los criterios sugeridos por el Profesor Robert Dahl. Las situaciones sugeridas son las siguientes:

- Una violación de la integridad de las instituciones centrales y/o de los controles constitucionales internos que permitan la separación de los poderes.

- La celebración de elecciones que no cumplan con normas internacionales mínimas.

- La alteración de la periodicidad de las elecciones o el hecho de que no se respeten sus resultados.

\footnotetext{
Véase CP/Doc. 4184/07.

Carter, Jimmy; (2005) conferencia sobre Problemas y Peligros de la Democracia, Cátedra de las Américas OEA: «Una Lección de Democracia», Universidad San Martín de Porres, pp. 26 y ss.
} 
- La violación sistemática de las libertades fundamentales (expresión, asociación, derechos de las minorías).

- La interrupción anticonstitucional de un mandato.

- La interferencia arbitraria o ilegal en los nombramientos o deliberaciones de los otros poderes del Estado.

- El uso sistémico de cargos públicos para silenciar, hostigar o interrumpir las actividades normales y legales de los miembros de la oposición política, de la prensa o de la sociedad civil.

Estas hipótesis requieren ciertamente de mayor precisión, junto con una apreciación política.

Las respuestas que se den a estas interrogantes serán cruciales en la evolución de la CDI e influirán en la evaluación que la comunidad internacional tenga de la eficacia de la OEA.

Creemos que, en este orden de ideas, será necesario reflexionar sobre algunos puntos:

1. Utilizar la CDI como instrumento que permita identificar los déficits de la democracia en la región y promover modelos para asistir a los países en el fortalecimiento de los regímenes democráticos. No estamos pensando en imposiciones o sanciones, sino en abrir espacios para una cooperación activa en esta esfera. El Secretario General, en su informe de $2007^{8}$, se refirió a este punto y expuso algunas opciones para conocer el estado de la

8 Ibid. Nota 3 supra. democracia en el hemisferio. Concretamente, sugirió la evaluación sectorial de los elementos constitutivos de la democracia a través de los informes que la Secretaría elabora en áreas como derechos humanos, corrupción, observación electoral, violencia contra la mujer y discapacidad. El objetivo sería utilizar esa información con criterio preventivo para establecer un diálogo con los países pertinentes con miras a mejorar la calidad de la democracia.

2. Ampliar la posibilidad de invocar la CDI por otros poderes del Estado.

3. Reforzar la capacidad de iniciativa del Secretario General en el marco de un multilateralismo moderno que no se interprete como una supranacionalidad. Así, el Secretario General podría actuar de oficio en los casos en que existe una amenaza a la democracia y ejercería las facultades que le confiere el artículo 110 inciso 2 de la Carta de la OEA. Ello puede revitalizar la acción del Sistema.

4. Establecer un diálogo oportuno, que haga las veces de "alerta temprana» entre la Comisión Interamericana de Derechos Humanos (CIDH), que goza de autonomía e independencia, los órganos políticos y la Secretaría General, respecto de situaciones en que la $\mathrm{CIDH}$ prevea una crisis que pueda amenazar la democracia y los derechos humanos. Atendiendo a ese principio, la coordinación con los órga- 
nos políticos es indispensable para dar eficacia al proceso.

5. Estudiar una revisión de la Carta. El actual Presidente de la Comisión de Asuntos Jurídicos y Políticos está interesado en trabajar esta materia pero debe verse si hay ambiente político para ello. El Comité Jurídico Interamericano ha hecho un estudio analítico recientemente expuesto ante la $\mathrm{CJI}^{9}$ en el que se hace referencia a diversos desafíos y se examina la bibliografía especializada relativa a la CDI.

6. Establecer mecanismos para facilitar la solución de conflictos interinstitucionales. La Organización tiene capacidad para ser un espacio de intercambio de experiencias constitucionales y legales, y puede profundizar una acción en esa área. Se trata de un trabajo preventivo frente a situaciones que puedan amenazar el orden democrático.

7. La OEA se ha constituido en agente para asistir en el perfeccionamiento de los sistemas electorales y en un referente de la observación de elecciones. Es indispensable continuar dando apoyo político y material a esta labor que permite implementar la CDI (Capítulo V) y consolidar la legitimidad de origen de la democracia. La elaboración de estándares electorales es una contribución efectiva al mejoramiento de la calidad

9 CJI/Doc. 317/09/corr.l «Seguimiento de la Aplicación de la Carta Democrática Interamericana» OEA, 19 de marzo de 2009. de nuestros sistemas democráticos.

8. Implementar el capítulo de prevención. La OEA está analizando el diseño de una agenda democrática, lo que ya fue sugerido en la V Cumbre de las Américas. Ello puede contribuir a asegurar mejores condiciones de sustentabilidad democrática.

\section{DeRECHOS HUMANOS}

El Sistema Interamericano de Derechos Humanos (SIDH) tiene una característica central, consistente en que los gobiernos han dotado a sus dos instancias principales, la Comisión y la Corte, de autonomía e independencia.

La Comisión (CIDH) actúa en materia de prevención, supervisión y protección. La Corte es un órgano jurisdiccional, cuyas sentencias son obligatorias para los Estados que han reconocido su competencia.

Recientemente, en la conmemoración del cincuentenario de la CIDH, se recordó que el aporte del SIDH en sus primeras décadas, se centró en la protección frente a violaciones masivas y sistemáticas de los derechos humanos. Posteriormente, se han identificado otros temas abordados en la etapa más reciente: libertad de expresión, acceso a la información, igualdad de género, derechos de los pueblos indígenas, discriminación y desarrollo progresivo de los derechos económicos, sociales y culturales (DESC).

El Sistema ha contribuido en tres áreas esenciales: 
1. Desarrollo de un cuerpo normativo que consagra estándares internacionales:

- Convención Americana sobre Derechos Humanos

- Protocolo de San Salvador sobre los derechos económicos, sociales y culturales

- Protocolo sobre la abolición de la pena de muerte

- Otras Convenciones Interamericanas

- La prevención y sanción de la tortura

- La desaparición forzada de personas

- Prevención, sanción y erradicación de la violencia contra la mujer (Belem do Pará)

- La eliminación de todas las formas de discriminación contra las personas con discapacidad.

2. Procesos normativos internos. La implementación efectiva de las decisiones de los órganos del SIDH es un elemento central de la protección regional. Por otra parte, la reparación de las violaciones es una forma de tutelar aquello a que los países se comprometieron al ratificar la Convención Americana sobre Derechos Humanos y otros instrumentos. Es necesario consignar que las sentencias dictadas por la Corte (artículos 67 y 68 de la Convención) tienen carácter definitivo, inapelable y vinculante. En el caso de la Comisión, las medidas cautelares, aunque no tienen rango convencional, emanan de los poderes entregados por el Estatuto y de una práctica sucesiva; en cuanto a los informes de solución amistosa aceptados por las partes, se entiende que ellos gozan de carácter vinculante. Los informes definitivos o de fondo de la Comisión en casos contenciosos generan para los Estados una obligación de cumplirlos de buena fe.

La jurisprudencia de la Corte Interamericana de Derechos Humanos ha sido decisiva en este proceso:

- Caso Barrios Altos contra Perú. Dejó sin efecto las leyes de amnistía. Permitió que se reabrieran miles de causas penales ${ }^{10}$.

- Caso de la Comunidad Awas Tingui contra Nicaragua. Se ordenó al Estado elaborar un marco jurídico que permitiera delimitar las propiedades indígenas. El Estado lo hizo y muchas comunidades han podido solucionar sus problemas ${ }^{11}$.

- Caso Olmedo Bustos (La Ultima Tentación de Cristo) contra Chile. Se ordenó al Estado que reformara su Constitución Política en materia de censura cinematográfica para dejarla acorde con la Convención Americana de Derechos Huma-

10 Corte I.D.H., Caso Barrios Altos vs. Perú. Fondo. Sentencia de 4 de marzo de 200. Serie C No. 75; Corte I.D.H., Caso Barrios Altos vs. Perú. Reparaciones y Costas. Sentencia de 30 de noviembre de 2001. Serie C No. 87.

11 Corte I.D.H., Caso de la Comunidad Mayagna (Sumo) Awas Tingni vs. Nicaragua. Fondo, Reparaciones y Costas. Sentencia del 31 de agosto de 2001. Serie C. $\mathrm{N}^{\circ} 79$. 
nos. El Estado cumplió y reformó la Constitución ${ }^{12}$.

- Caso Claude Reyes contra Chile. Se ordenó al Estado que creara un marco jurídico y un mecanismo que permitiera el acceso a la información pública. El Estado dictó la ley de información pública y creo el Consejo de la Transparencia ${ }^{13}$.

- Caso Herrera Ulloa contra Costa Rica. Se ordenó al Estado que tomara las medidas pertinentes para permitir una revisión integral de la sentencia de primera instancia en materia penal. El Estado dictó una ley que fijó las causales por las cuales procede el recurso de casación ${ }^{14}$.

3. Una variedad de documentos consagran y reafirman el valor de los derechos humanos. A su vez, un conjunto de declaraciones políticas bilaterales, multilaterales, regionales, subregionales y, recientemente, la Declaración de Compromiso de la V Cumbre de las Américas, confirman la prioridad política de esta materia. El tema de fondo es que ello se traduzca en un

12 Corte I.D.H., Caso «La Última Tentación de Cristo" (Olmedo Bustos y otros contra Chile). Fondo, Reparaciones y Costas. Sentencia de 5 de febrero de 2001. Serie C No. 73.

13 Corte I.D.H., Caso Claude Reyes y otros contra Chile. Fondo, Reparaciones y Costas. Sentencia del 9 de septiembre de 2006. Serie C No. 151.

14 Corte I.D.H., Caso Herrera Ulloa contra Costa Rica. Excepciones Preliminares. Fondo, Reparaciones y Costas. Sentencia de 2 de julio de 2004. Serie C. No. 107. sistema universal en que los Estados asignen mayores recursos a los órganos del SIDH y en la profundización de un diálogo constructivo entre todos los usuarios. Estas preocupaciones fueron recogidas en la Declaración de Santiago, conmemorativa del cincuentenario de la CIDH (Santiago de Chile, 2009).

Estas referencias permiten concluir que este tema plantea desafíos jurídicos, políticos y éticos, así como un trabajo sustantivo, especialmente en las instancias competentes de los países miembros. Se busca asegurar que las obligaciones asumidas internacionalmente sean cumplidas y que el derecho interno se ajuste a dichas normas. El artículo 2 de la Convención Americana obliga expresamente a los Estados a adoptar a nivel local aquellas medidas de carácter legislativo, judicial o administrativo que fueren necesarias para hacer efectivos los derechos consagrados convencionalmente. Para fortalecer la implementación de las decisiones y sentencias del SIDH es indispensable una estrategia que involucre a diversos actores a fin de garantizar una protección jurídica efectiva. El Centro para la Justicia y el Derecho Internacional (CEJIL) ha analizado con profundidad este tema, contribuyendo al desarrollo progresivo del derecho internacional de los derechos Humanos pero, por sobre todo, generando conciencia pública y política sobre una dimensión esencial de las democracias ${ }^{15}$.

15 Krsticevic, Viviana; (2007) «Reflexiones sobre la Ejecución de las Decisiones del Sistema Interamericano de Protección de los Derechos Humanos, Jurisprudencia, 
Destaquemos algunos aspectos que deberían abordarse:

El desarrollo de un capítulo preventivo en materia de derechos humanos es un desafío que debe entenderse como parte de la cultura democrática. Se trata de un proceso que requiere de atención política y pedagógica cotidiana para reforzar la legítima preocupación de la comunidad internacional y fortalecer la cooperación en este ámbito.

Los derechos humanos son incorporados en el discurso político pero la realidad muestra que la labor de cautela produce tensiones entre los órganos del Sistema y los gobiernos. Esto se manifiesta especialmente en las visitas in loco y en los informes de la CIDH.

Un aspecto fundamental y visible en materia de derechos humanos es el papel de la sociedad civil. Esto se vincula a un tema de principios, aunque esencialmente se trata de una cuestión de acceso y de defensoría de las víctimas.

El SIDH requiere, por tanto, de perfeccionamientos, en particular en términos de recursos, en la agilización de los procedimientos y mecanismos de implementación de sus decisiones. En 2008 la CIDH recibió 1323 denuncias, de las cuales se dio trámite a 118 . Sabemos que se están estudiando medidas para responder a este problema. Se trata aquí de un punto político

Normativa y Experiencias Nacionales, Washington, D.C, pp 5 y ss. que requiere de atención y respuesta urgentes, ya que de otra manera este órgano quedaría limitado para una acción efectiva y planificada dentro del funcionamiento democrático.

\section{Seguridad Multidimensional}

Este tema se ha agregado al concepto clásico de seguridad estatal, para abarcar la seguridad centrada en las personas.

La Organización continúa trabajando en el ámbito de las medidas de fomento de la confianza, asociadas a la visión tradicional. Lamentablemente, los países de la región han entregado escasa información en esta materia y se requiere darle una mayor prioridad política. En 2007 solo tres Estados enviaron informes a la Comisión de Seguridad Hemisférica ${ }^{16}$.

Nuevos actores, problemas y amenazas se expresan en el enfoque multidimensional de seguridad consagrado en la Asamblea General celebrada en Barbados (2002) ${ }^{17}$.

El desafío de hoy es responder a los fenómenos vinculados con el crimen organizado, el narcotráfico, el acceso a armas pequeñas y ligeras de actores

16 «Catálogo de Informes de los Estados Miembros Presentados de Conformidad con las Resoluciones de la Asamblea General en Materia de Seguridad Hemisférica 1997-2009» (doc.CP/CDH/INF. 1128/09, de 3/7/2009).

17 «Declaración de Bridgetown», Asamblea General de la OEA, doc. AG/DEC.27 (XXXII-O/02). 
no estatales y otros ilícitos de carácter trasnacional. Estas realidades generan violencia e inseguridad, cuestionan el monopolio legítimo de la fuerza por parte del Estado, afectando la calidad de vida de los ciudadanos y, lo que es más grave, el Estado de derecho y la institucionalidad democrática.

En nuestra región los homicidios duplican el promedio mundial, llegando en algunas zonas a quintuplicarlo. No obstante que en las Américas solo habita el $8 \%$ de la población mundial, ocurre en ellas el $42 \%$ de los homicidios con armas de fuego y el $66 \%$ de los secuestros del mundo ${ }^{18}$.

En este contexto, dentro del marco interamericano se ha trabajado para implementar la Declaración de Seguridad para las Américas, aprobada en México en 2003, en torno a cinco pilares: gestión de la seguridad pública; prevención de la delincuencia, la violencia y la inseguridad; gestión de la policía; participación ciudadana y comunitaria; y cooperación internacional.

En la Secretaría Política de la OEA se ha venido desarrollando el concepto de la nueva estatalidad. El término no se relaciona con el viejo estatismo, que tiene connotaciones más ideológicas o políticas. Lo que se busca es un nuevo

18 Discurso del Secretario General de la OEA, José Miguel Insulza, en la Conferencia Especializada Interamericana sobre Seguridad Pública: Reunión de Expertos Preparatoria de la MISPA II, Montevideo, Uruguay - 4 de agosto de 2009 (doc. MISPA/RE/INF.8/09, de 4/8/2009).
Estado para una nueva democracia, de mejor calidad, un Estado con capacidad real de garantizar a los ciudadanos el acceso a bienes públicos básicos. La seguridad pública es uno de ellos. Algunos ámbitos que deben considerarse:

- El respeto de los derechos humanos.

- La ampliación de las funciones de las fuerzas armadas. Esta tendencia debe ser observada con detenimiento, porque es una esfera inherente a las policías. Sin duda, este es un punto sobre el cual los regímenes democráticos deben reflexionar y actuar con prudencia, dadas las superposiciones que pueden darse entre las competencias de ambas instituciones ${ }^{19}$.

- Los niveles de desarrollo e inclusión que debe tener una sociedad una de las regiones de mayor desigualdad, ¿Cómo se pueden mejorar los índices de seguridad?

Cabe preguntarse la contribución que pueden hacer la OEA o el sistema multilateral

- Fortalecer las capacidades institucionales y los recursos humanos policiales y civiles para definir e implementar políticas públicas en materia de seguridad. Se está preparando un estudio de factibilidad sobre las me-

19 Véase Rojas Aravena, Francisco, "América Latina: Defensa y Seguridad en el Siglo XXI», Documento preparado para la Reunión de RESDAL, "La Situación de la Defensa en América Latina y las Perspectivas a Futuro", Bolivia, julio de 2009, pp 37 y ss. 
jores formas de reforzar en la región el entrenamiento y la formación de personal con responsabilidad en seguridad pública ${ }^{20}$. Esta idea parece esencial para una gestión integral del tema y viene de un mandato de la Primera Reunión Ministerial de Seguridad Pública (MISPA) analizado en la MISPA II en República Dominicana (2-5 noviembre de 2009). Se trata de una preocupación política prioritaria para la región.

- Crear un observatorio interamericano de seguridad para analizar y divulgar información comparable sobre crimen y violencia. Ello puede contribuir a un mejor diseño de políticas públicas en seguridad, monitoreo y desarrollo de indicadores.

- Con este objetivo el BID y la Universidad del Valle en Colombia elaboran actualmente un proyecto para establecer parámetros comparables en materia de seguridad pública.

- La OEA, como cuerpo político, puede proponer estrategias innovadoras y alianzas con otros organismos del sistema interamericano y de las Naciones Unidas, para potenciar las capacidades de luchar contra estos ilícitos vinculados a la seguridad pública.

20 Estudio de Factibilidad para el Fortalecimiento en la Región de la fomación del personal con responsabilidad en temas de Seguridad Pública (MISPA/RE/doc.4/09. 04 agosto 2009)

\section{DESARRollo INTEGRAL}

- En la OEA se ha continuado abriendo un espacio político para los temas de desarrollo enunciados en el Capítulo VII de la Carta titulado «Desarrollo Integral». La idea es complementar el enfoque social de manera de expresar la relación entre el desarrollo integral, la superación de la pobreza y la gobernabilidad democrática, como una prioridad en la agenda interamericana. ¿Cómo está trabajando este punto la Organización?

- En la negociación de una Carta Social. Ese proceso busca desarrollar garantías explícitas en materia de derechos económicos, sociales y culturales (educación, salud, empleo, cultura, ciencia y tecnología). Debemos reconocer y esto hay que decirlo, que el enfoque expresando, que algunos Estados promovemos, genera dificultades, no porque se desconozca esta categoría sino por un problema de exigibilidad, vinculado más bien a diferentes sistemas jurídicos. Este aspecto ha sido analizado en el proceso de laboración del Protocolo Facultativo al Pacto Internacional de Derechos Económicos, Sociales y Culturales. Al respecto, el Profesor Víctor Abramovich sostiene que los Estados o los sujetos obligados deben tener un amplio espacio de discrecionalidad para elegir las medidas específicas, destinadas a hacer efectivos estos derechos ${ }^{21}$. Desde esa pers-

21 Abramovich, Víctor; (2006) «Una Aproximación al Enfoque de Derechos en las 
pectiva, sería esencial que esta concepción no se constituya en una limitante para quienes definen politicas y estrategias de desarrollo. Es probable que la propia aplicación de estos derechos permita ir buscando soluciones que faciliten el desarrollo progresivo de esta categoría.

- Para muchos la Carta Social es el complemento de la Carta Democrática Interamericana (CDI), lo cual parece una afirmación políticamente correcta. La propia CDI así lo reconoce al señalar en su Capítulo III que la democracia y el desarrollo económico y social son interdependientes y se refuerzan mutuamente. La CDI proclama la ciudadanía social, que constituye una dimensión fundamental para reforzar la democracia. Esta es una base para la expansión de los derechos sociales. Sin ella, la democracia puede debilitarse y afectar la adhesión ciudadana, lo que es peligroso en una región con altos índices de pobreza e inequidad.

- La prioridad política en esta área es el lanzamiento de la Red Interamericana de Protección Social, dando seguimiento así a una iniciativa propuesta por Chile, aprobada en la Primera Reunión de Ministros y Altas Autoridades de Desarrollo Social (Reñaca, 2008) y ratificada por la V Cumbre de las Américas. Ese fue un punto de inflexión al reconocerse colectivamente que el fortalecimiento de la dimensión social de las políticas

Estrategias y Políticas de Desarrollo»; Revista de la CEPAL 88, abril. públicas es esencial para la gobernabilidad democrática.

- Es claro que no hay modelos ni experiencias únicas en el ámbito del desarrollo social y que es posible un trabajo cooperativo, en que la OEA juegue un papel articulador. Esta es la lógica con la cual se han socializado experiencias como Chile Solidario, Bolsa Familia de Brasil, Vivir Mejor de México, Oportunidades de Estados Unidos, y Step-up de Trinidad y Toba$\mathrm{go}^{22}$.

- Un aspecto más general, y que relaciona el Sistema Interamericano con el global, es cómo la OEA puede contribuir a generar en la región las condiciones que permitan dar cumplimiento a los Objetivos de Desarrollo del Milenio.

- La formación y perfeccionamiento del capital humano para mejorar la competitividad de la región. Esto tiene que ver con educación (becas), ciencia y tecnología, sobre una base de no discriminación e inclusión.

- La OEA debe continuar siendo un espacio de cooperación para el intercambio de buenas prácticas en materia de políticas públicas sociales y el reforzamiento de las instituciones nacionales en esta área. Si la Organización es entendida en esa línea y no como una agencia de desarrollo, po-

22 Véase Gacitúa-Marió, Estanislao, Andrew Norton y Sophia V. Georgieva, «Building Equality and Opportunity Through Social Guarantees» (2009), en New Approaches to Public Policy and the Realization of Rights,Banco Mundial . 
Pedro Oyarce • La oea y la cooperación hemisférica

dremos contribuir a que la OEA ella utilice sus ventajas comparativas y haga un aporte concreto al multilateralismo en el capítulo social.

\section{Conclusiones}

Estas reflexiones sitúan a la OEA como un actor más cercano a temas que preocupan a la gente y si bien a veces los avances son limitados ello se debe a que los gobiernos no están en condiciones de adoptar decisiones políticas. El mecanismo del consenso es percibido como un patrimonio de esta institución que, junto con sus ventajas, puede ser un obstáculo para abordar temas políticos urgentes que requieren de una capacidad de reacción rápida pero en que las diferencias de opinión son difícilmente superables. En situaciones críticas, algunos países han planteado que esta forma de tomar decisiones puede transformarse en una suerte de veto y paralizar a la Organización. No creemos que exista una voluntad para cambiar esta práctica.

Sin duda, los capítulos más sensibles y determinantes en la evaluación de la OEA se vinculan con la defensa de la democracia y los derechos humanos. Este trabajo sistematiza, por tanto, un conjunto de problemas que merecen ser analizados en el seno de los cuerpos políticos.

La OEA es capaz de influir en los ámbitos internos e internacionales, donde los conceptos de soberanía y no intervención siguen siendo funda- mentales. Sin embargo, ellos no pueden ser invocados para rechazar la legítima preocupación de la comunidad internacional en materias relativas a la dignidad humana y a la democracia. La OEA tiene que preservar una lógica constructiva que lleve a superar las tradicionales reticencias que plantean estos temas.

La Organización tiene además una fuerte presencia en la definición de políticas públicas y ello es reflejo de un trabajo sistemático, respetado y demandado. La OEA es también un foro efectivo de cooperación interamericana y un espacio privilegiado para proyectar la prioridad regional de la política exterior de los países miembros. Es el resultado de un entendimiento de cómo funciona un sistema multilateral moderno, que comprenda la prevención, la interlocución entre sus miembros y, cuando sea necesario, la sanción.

Por último, es indispensable insistir en que la OEA debe seguir siendo el lugar donde se custodien los principios y valores constitutivos de las sociedades interamericanas. Esto es central en una región donde el respeto al derecho internacional es un pilar fundamental de la cultura política.

El suministro de los recursos humanos y financieros adecuados es también una obligación política hacia la OEA, la que no podrá continuar su acción cooperativa si el presupuesto ordinario no se lo permite. En tal sentido, las contribuciones voluntarias ayudan pero la institución requiere de una mejor previsión presupuestaria. 